\title{
Impacto da crise econômica brasileira no transporte aéreo no país: Uma análise da influência do PIB e da inflação
}

\author{
Gustavo J. L. Coppio ${ }^{1}$, Mauro Caetano ${ }^{2}$, Cláudio J. P. Alves ${ }^{3}$, Luciana S. Cividanes ${ }^{4}$, \\ Maryangela G. Lima ${ }^{5}$
}

${ }^{1}$ Instituto Tecnológico de Aeronáutica, São José dos Campos, SP, Brasil, gustavocoppio@gmail.com

2 Universidade Federal de Goiás, Goiânia, GO, Brasil, maurocaetano1912@gmail.com

${ }^{3}$ Instituto Tecnológico de Aeronáutica, São José dos Campos, SP, Brasil, claudioj@ita.br

${ }^{4}$ Instituto Tecnológico de Aeronáutica, São José dos Campos, SP, Brasil, lu_civi@yahoo.com.br

${ }^{5}$ Instituto Tecnológico de Aeronáutica, São José dos Campos, SP, Brasil, magdlima@gmail.com

\section{Recebido:}

02 de setembro de 2016

Aceito para publicação:

21 de março de 2017

Publicado:

31 de agosto de 2017

Editor de área:

Li Weigang, UnB

\section{Palavras-chaves:}

PIB.

Inflação.

Movimentação de passageiros

aéreos.

Brasil.

\section{Keywords:}

GDP.

Inflation.

Handling of air passengers.

Brazil.

DOI:10.14295/transportes.v25i2.1241

\section{RESUMO}

A correlação entre movimentação de passageiros e economia é fundamental para a previsão da demanda por transporte aéreo, sendo que a relação entre o Produto Interno Bruto (PIB) e a movimentação de passageiros tem sido comumente explorada pela literature em previsões. A inflação, entretanto, consiste em uma variável que pode estar associada à movimentação de passageiros e que não tem sido explorada. Como o Brasil tem enfrentado crises econômicas desde 2014, o objetivo deste estudo consiste em verificar a influência do PIB e da inflação na movimentação de passageiros aéreos do país, utilizando metodologia baseada em regressão linear, para o período de 2013 a 2015, considerando sete aeroportos do Brasil. Os resultados demonstram uma redução na movimentação de passageiros no transporte doméstico e internacional, acompanhada da queda do PIB e aumento da inflação para todos os aeroportos estudados, com exceção do Aeroporto Internacional de Brasília, maior hub doméstico do Brasil atualmente. Os resultados também demonstram melhor ajuste das curvas para a utilização de PIB do que inflação e uma redução nas tarifas e na quantidade de assentos ofertados no transporte aéreo doméstico. Com as equações obtidas, são realizadas previsões para a movimentação de passageiros no transporte aéreo para 2016. $\mathrm{O}$ artigo tem como base $\mathrm{o}$ ano de 2016, mas a metodologia proposta pode ser utilizada para outros anos.

\section{ABSTRACT}

The correlation between air passenger transport and the economy is crucial for predicting the demand for air transport, and the relationship between the Gross Domestic Product (GDP) and air passenger transport has been commonly exploited by literature forecasts. Inflation, however, consists of a variable that can be associated with air passenger transport and has not been exploited. As Brazil has faced economic crises since 2014, the aim of this study is to verify the influence of GDP and Inflation in the air passenger transport of the country, using a linear regression methodology for the period from 2013 to 2015, considering seven airports in Brazil. The results show a reduction of domestic and international air passenger transport, together with the fall in GDP and rising of inflation for all airports studied, with the exception of Brasilia International Airport, the largest domestic hub currently Brazil's. The results also demonstrate best fit curves for the use of GDP than inflation and a reduction in tariffs and the number of seats offered on domestic air transport. With the equations obtained, forecasts for air passenger transport for 2016 are performed. The article is based on the year 2016, but the methodology can be used for other years. 


\section{INTRODUÇÃO}

A relação entre o aumento da demanda por transporte aéreo e o crescimento da economia tem sido discutida na literatura para o melhor entendimento do rápido crescimento do transporte aéreo no mundo e, em especial, no Brasil. Entretanto, a crise financeira do país, que se iniciou em 2014, alterou esta situação de crescimento do setor aéreo brasileiro. Em resposta à alteração da demanda por transporte aéreo no Brasil, as companhias aéreas também modificaram as tarifas das passagens, assim como a quantidade de assentos ofertados. Entender a dinâmica dessas alterações é essencial para a previsão da demanda por transporte aéreo e planejamento de investimentos no setor (Marazzo et al., 2010).

De uma forma geral, o Produto Interno Bruto (PIB) é a variável econômica utilizada no estudo das mudanças na movimentação de passageiros aéreos. Entretanto, os estudos, são, em geral, realizados para um longo período de análise (entre 15 e 35 anos). Para curtos períodos (até cerca de 7 anos), a relação entre crescimento do PIB e aumento da movimentação de passageiros não é tão direta, e outras variáveis podem ser consideradas para uma melhor avaliação e previsão da demanda por transporte aéreo em um futuro próximo (Hu et al., 2015). Na maioria dos trabalhos relacionados ao tema, os dados de PIB são utilizados após a correção da inflação. Ou seja, os dados de inflação estão contidos nos valores de PIB. Desta forma, a própria inflação pode estar correlacionada à movimentação de passageiros aéreos. Assim, a Inflação é uma variável econômica que está associada à crise econômica e tem potencial para ser utilizada neste tipo de estudo, apesar de ter sido pouco explorada pela literatura (Dobruszkes e Van Hamme, 2011).

Além disso, o efeito da crise econômica brasileira na movimentação de passageiros aéreos pode ser diferente dependendo do tipo de transporte aéreo analisado, se doméstico ou internacional, e ainda pode depender do aeroporto analisado. Estudos da influência de crise financeira de um país na movimentação de passageiros aéreos são realizados, em geral, apenas para o transporte doméstico, uma vez que este será, obviamente, mais influenciado pela crise daquele país (Rimmer, 2000; Dobruszkes e Van Hamme, 2011). Entretanto, a movimentação de passageiros no transporte aéreo internacional também pode ser influenciada pela crise do país. Por fim, a literatura apresenta, em geral, estudos da movimentação média de passageiros aéreos de todos os aeroportos de um país, o que pode não representar bem um aeroporto em específico. Por exemplo, enquanto um aeroporto pode ter tido redução da movimentação de passageiros, outro pode ter mostrado aumento, e a média, neste caso, pode não mostrar nenhuma alteração, mascarando os resultados.

Desta forma, o objetivo deste estudo consiste em estudar a influência de duas variáveis econômicas, PIB e Inflação, na movimentação de passageiros no transporte aéreo doméstico e internacional de sete aeroportos do Brasil, incluindo o principal deles em termos de movimentação anual de passageiros, o Aeroporto Internacional de São Paulo/Guarulhos, e ainda um aeroporto de cada uma das cinco regiões em que se divide o território brasileiro: Norte, Nordeste, Centro-Oeste, Sudeste e Sul. Assim, foi possível apresentar equações de previsão de transporte aéreo para cada um dos aeroportos estudados.

\section{CRISE ECONÔMICA E TRANSPORTE AÉREO}

Costuma-se frequentemente argumentar que a atividade do transporte aéreo de passageiros está relacionada com a atividade econômica, em especial com o Produto Interno Bruto (PIB) (Profillidis e Botzoris, 2015). Marazzo et al. (2010), por exemplo, investigaram a relação entre PIB e movimentação de passageiros aéreos entre 1966 e 2006 no Brasil e concluíram que estas variáveis são cointegradas, mostrando uma forte relação positiva da movimentação de passageiros com uma mudança positiva no PIB. De acordo com Marazzo et al. (2010), se pelo menos duas séries são não-estacionárias e apresentam um caminho semelhante ao longo do tempo, pode haver alguma combinação linear entre elas que é estacionária. Quando isso acontece, diz-se que essas variáveis são cointegradas.

No entanto, o PIB reagiu a uma mudança na movimentação de passageiros de um modo mais lento e moderado. Chi e Baek (2013) sugeriram que, a longo prazo, tanto o tráfego aéreo de passageiros como o de mercadorias tendem a aumentar com o crescimento econômico, o que não ocorre a curto prazo com 
o transporte de mercadorias. Profillidis e Botzoris (2015) demonstraram uma relação causal entre a atividade de transporte aéreo de passageiros e o PIB, sendo as correlações mais sólidas para mercados aéreos mais maduros, sugerindo uma taxa de crescimento mundial do transporte aéreo de passageiros de 3,8\% para os próximos 15 anos.

A correlação entre movimentação de passageiros e economia tem sido comumente estudada para a previsão da demanda por transporte aéreo de passageiros e para o planejamento da necessidade de investimentos no setor. Uma previsão inadequada pode levar à congestão do sistema e ineficiência dos serviços. Por outro lado, estimativas muito altas de demanda podem produzir um excesso de capacidade de infraestrutura e custos desnecessários (Marazzo et al., 2010).

Grande parte dos estudos utiliza o PIB como variável econômica para prever a demanda por transporte aéreo. Porém, de acordo com Hu et al. (2015), o PIB pode não ser uma boa variável para esta previsão a curto prazo. Desta forma, outras variáveis econômicas poderiam ser utilizadas. Assim como o PIB, outra variável determinante da economia de um país, em uma análise macroeconômica, é a Inflação, que mede a taxa de aumento contínuo e generalizado do nível geral de preços e está associada à estabilidade econômica do país. O PIB e a Inflação impactam diretamente em diversos setores da economia, como no mercado de bens e serviços, mercado de trabalho, monetário, de títulos e de divisas (Vasconcellos e Garcia, 1998). Sendo assim, a Inflação poderia ser utilizada na previsão da movimentação de passageiros, com o objetivo de melhorar as previsões de demanda a curto prazo.

O Brasil tem enfrentado crises econômicas desde 2014, com sucessivas quedas do PIB real e aumento da inflação. Essas crises surgiram após um período de crescimento econômico, investimentos em infraestrutura aeroportuária (Scarpel, 2013) e tarifas aéreas mais acessíveis (Wadud, 2015).

Uma crise financeira pode influenciar a atividade de transporte aéreo de uma forma não tão direta como a relação entre o crescimento econômico e o aumento do PIB, como foi mostrado por Rimmer (2000), que estudou o efeito da crise asiática de 1997 e 1998 no tráfego aéreo do Sudeste Asiático e entre a região e outras partes do mundo. Dobruszkes e Hamme (2011) mostraram efeitos da crise mundial no transporte aéreo, sendo que a mudança na oferta de assentos dependeu do país estudado.

A influência da crise econômica brasileira iniciada em 2014 na movimentação de passageiros do país foi pouco estudada pela literatura. Por exemplo, algumas variáveis que influenciam a movimentação de passageiros aéreos já estudadas pela literatura, além do PIB, são: evolução da população (Profillidis e Botzoris, 2015; Sivrikaya e Tunç, 2013); custo da tarifa aérea e preço do combustível aéreo (Wadud, 2014; Wadud, 2015); desenvolvimento da rede de transportes e custos de impostos sobre a aviação (Ashley, Hanson e Veldhuis, 1995; Vega e Reynolds-Feighan, 2016); distância entre destino de partida e de chegada e tempo de viagem (Sivrikaya e Tunç, 2013; Wu, Han e Hayashi, 2011); investimento estrangeiro direto (Kalayci e Yanginlar, 2016).

Além disso, a Inflação é uma variável que pode influenciar a movimentação de passageiros a curto prazo e que não foi explorada por outros estudos já publicados. Sendo assim, este estudo pretende correlacionar a queda do PIB e o aumento da Inflação no Brasil com a taxa de movimentação de passageiros no transporte aéreo, considerando o cenário brasileiro de crise econômica e também os recentes investimentos no setor aeroportuário no país, em especial para a Copa do Mundo de Futebol FIFA 2014 e Olimpíadas 2016. Estes investimentos somam R \$ 6,3 bilhões, de acordo com o Portal da Transparência.

Este trabalho apresenta equações de previsão de transporte aéreo para cada um dos aeroportos estudados, assim como a resposta das empresas aéreas brasileiras em relação às tarifas e assentos ofertados durante a crise, apresentando como contribuição acadêmica a análise de uma variável que não foi estudada pela literatura (Inflação) e a utilização de uma metodologia para estudo de curto prazo. Além disso, este estudo preenche outras lacunas relacionadas ao tema encontradas na literatura, como o estudo da influência da crise econômica brasileira tanto no transporte aéreo doméstico como no internacional, assim como o estudo de dados de sete principais aeroportos no país, e não uma média de dados dos aeroportos do país. Desta forma, ao preencher estas lacunas apontadas, este trabalho pretende melhorar as previsões de demanda a curto prazo da movimentação de passageiros aéreos no Brasil, durante a crise econômica, podendo a metodologia ser aplicada também para outros países e outros aeroportos. 
0 artigo tem como base o ano de 2016, porém a metodologia proposta pode facilmente ser aplicada para outros anos. Assim, os resultados deste estudo apresentam subsídios para um melhor entendimento da dinâmica das alterações do setor aeroportuário brasileiro a curto prazo, durante o período de crise econômica, para melhor planejamento do setor, assim como para a elaboração futura de modelos matemáticos para a determinação da evolução da movimentação de passageiros aéreos a longo prazo no Brasil, durante e após o período de crise econômica.

\section{MÉTODOS}

Para verificar a evolução da movimentação de passageiros aéreos do país durante a recente crise econômica, que teve início em 2014, foram usadas a mudança na movimentação de passageiros aéreos, como variável dependente, e a variação do PIB, como a principal variável independente.

Nesse estudo também é analisada a viabilidade do índice de inflação ser utilizado como uma variável independente. Tanto o PIB como a Inflação variam ao longo do tempo, assim como a movimentação de passageiros.

Dessa forma, foi realizada uma regressão linear dos dados (movimentação de passageiros versus PIB e movimentação de passageiros versus Inflação), conforme outros estudos relacionados ao tema (Profillidis e Botzoris, 2015; ICAO, 2006). Assim, foi possível realizar uma projeção de demanda futura por transporte aéreo no Brasil, em um cenário de crise econômica.

O estudo foi realizado para alguns dos principais aeroportos do país, de forma a englobar pelo menos um aeroporto de cada uma das cinco regiões do país: Norte, Nordeste, Centro-Oeste, Sudeste e Sul, com o objetivo de verificar se a crise afetou de forma diferenciada cada um destes aeroportos e regiões. Os sete aeroportos estudados foram: Aeroporto Internacional de São Paulo/Guarulhos (GRU), Aeroporto Internacional de Brasília (BSB), Aeroporto Internacional do Galeão (GIG), Aeroporto Internacional de Belém (BEL), Aeroporto Internacional de Salvador (SSA), Aeroporto Internacional de Goiânia (GYN), Aeroporto Internacional de Porto Alegre (POA).

0 estudo é apresentado tanto para movimentação de passageiros no transporte aéreo doméstico como no internacional. De uma forma geral, crises financeiras de um país estão mais associadas à movimentação de passageiros no transporte aéreo doméstico. Por isso, alguns estudos relacionados à influência da crise financeira na atividade econômica utilizaram apenas voos domésticos na análise (Rimmer, 2000; Dobruszkes e Van Hamme, 2011). Porém, comparar as semelhanças e diferenças da influência da crise econômica nos dois tipos de transporte, conforme realizado por Vega e Reynolds-Feighan (2016), torna-se necessário, de forma a possibilitar o planejamento das necessidades de investimentos no setor de transporte aéreo doméstico e no setor transporte aéreo internacional.

Os dados da movimentação de passageiros foram retirados da Empresa Brasileira de Infraestrutura Aeroportuária, Infraero, que é uma empresa pública federal brasileira que está entre as três maiores operadoras aeroportuárias do mundo, e das próprias operadoras dos aeroportos, como os de São Paulo (GRU), Brasília (BSB) e Rio de Janeiro (GIG), aeroportos que foram privatizados entre 2012 e 2013.

Os dados relativos ao PIB e à Inflação foram fornecidos pelo Instituto Brasileiro de Geografia e Estatística (IBGE). Os dados do PIB foram utilizados após correção da inflação, e por trimestre, uma vez que são fornecidos desta forma pelo IBGE. Assim, para a comparação, os dados relativos à movimentação de passageiros e à inflação também foram apresentados por trimestre. Os dados de Inflação que foram utilizados são aqueles relativos ao Índice Nacional de Preços ao Consumidor Amplo - IPCA.

Também foi estudado como as empresas aéreas brasileiras reagiram à crise, analisando o preço médio das tarifas aéreas domésticas no período e a quantidade de assentos ofertados, medidos em assentos quilômetros oferecidos (ASK; produto entre o número de assentos oferecidos e a distância das etapas). Estes dados foram coletados da Agência Nacional de Aviação Civil, ANAC.

Todas as informações que foram utilizadas são relativas ao período entre 2013 e 2015, compreendendo não apenas o período da crise, como também aquele imediatamente anterior à crise.

Assim, foi possível avaliar como a crise financeira brasileira influenciou a atividade do transporte 
aéreo no país para diferentes aeroportos e regiões, e como as empresas aéreas brasileiras responderam a estas mudanças, prevendo então a movimentação de passageiros no futuro, durante a crise econômica.

\section{ANÁLISE DE RESULTADOS}

A variação do PIB e da Inflação no Brasil foi analisada para o período entre 2013 e 2015, de acordo com os dados fornecidos pelo IBGE. A Figura 1 apresenta os dados coletados para a evolução do PIB e da Inflação por trimestre, considerando sua variação em relação ao mesmo trimestre do ano anterior, assim como os dados relativos às regressões lineares realizadas.

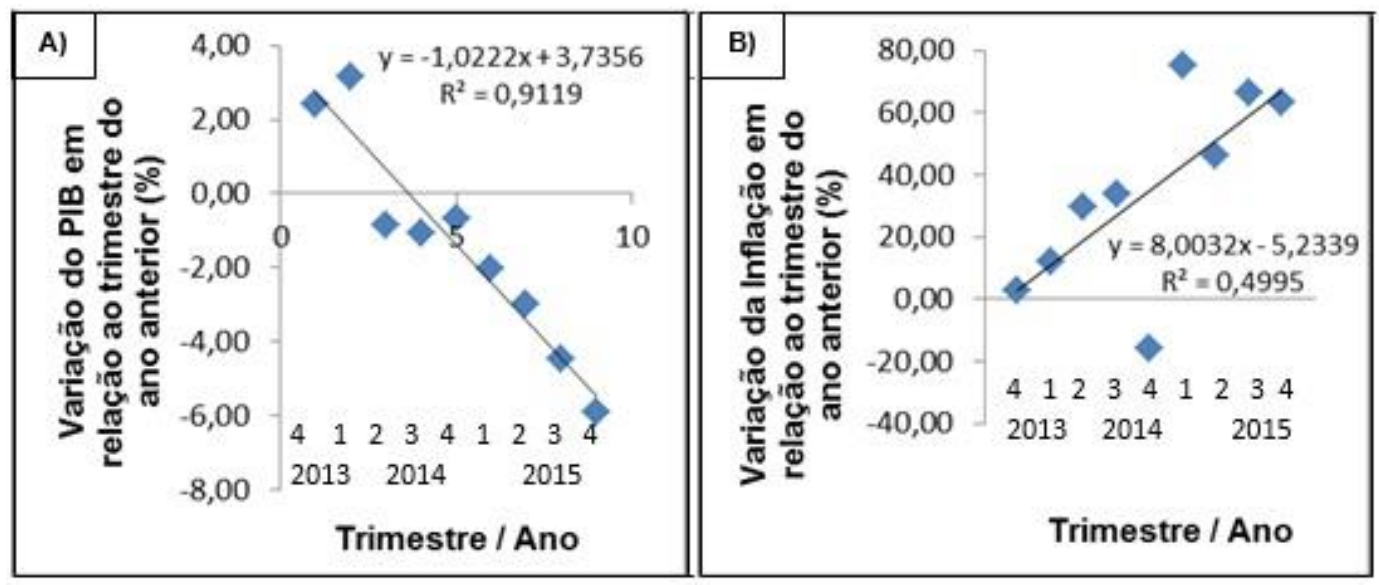

Figura 1. Evolução da variação do A) PIB e da B) Inflação no Brasil por trimestre (em relação ao mesmo trimestre do ano anterior), entre o quarto trimestre de 2013 e o quarto trimestre de 2015

Como pode ser observado pela Figura 1, PIB e Inflação variaram ao longo do tempo de forma oposta, sendo que os valores do PIB foram reduzindo e os valores da Inflação foram aumentando ao longo de praticamente todos os trimestres analisados. 0 coeficiente de correlação obtido foi de aproximadamente 0,9 para o PIB e de 0,5 para a Inflação. Uma das causas que explica o menor fator de correlação para a Inflação é a presença de um ponto discrepante da tendência e claramente fora da curva, como pode ser observado na Figura 1B. Este ponto corresponde ao quarto trimestre de 2014, quando a variação da Inflação foi de $-15,8 \%$.

Uma possível explicação para o ponto discrepante da Figura 1B é o término da Copa do Mundo de Futebol FIFA 2014, que ocorreu no Brasil entre junho e julho de 2014. Após a Copa, a Inflação teve um grande decréscimo, em especial devido à queda dos preços de passagens aéreas e tarifas hoteleiras (IBGE, 2014).

Para verificar a influência do ponto discrepante da Figura 1B nos dados relativos à Inflação, a equação da reta foi calculada para os dados de Inflação sem a presença deste ponto, e o resultado foi um coeficiente de correlação de aproximadamente 0,8 , valor consideravelmente maior do que o coeficiente de correlação de aproximadamente 0,5 da Figura 1B. Assim, apesar da presença do ponto fora da curva da Figura 1B, tanto o PIB como a Inflação são variáveis que seguiram uma tendência coerente com a crise econômica do Brasil no período analisado, ambas seguindo a equação de uma reta.

A movimentação de passageiros no transporte aéreo doméstico e internacional no Brasil durante a crise financeira foi estudada para sete aeroportos. Para cada um deles, foi realizada a regressão linear dos dados de movimentação de passageiros em relação ao tempo. A Figura 2 apresenta os resultados relativos ao Aeroporto Internacional de São Paulo/Guarulhos (GRU). As equações obtidas para este aeroporto são apresentadas na própria Figura 2 e na Tabela 1, que apresenta os resultados obtidos para todos os outros aeroportos aqui estudados.

A Figura 2 e a Tabela 1 demonstram queda na movimentação de passageiros tanto no transporte aéreo doméstico como no internacional para praticamente todos os aeroportos analisados. Apenas o Aeroporto Internacional de Salvador e o do Rio de Janeiro apresentaram aumento de movimentação de 
passageiros no transporte aéreo internacional no período analisado (coeficiente angular positivo para equação de PAX Int por trimestre).
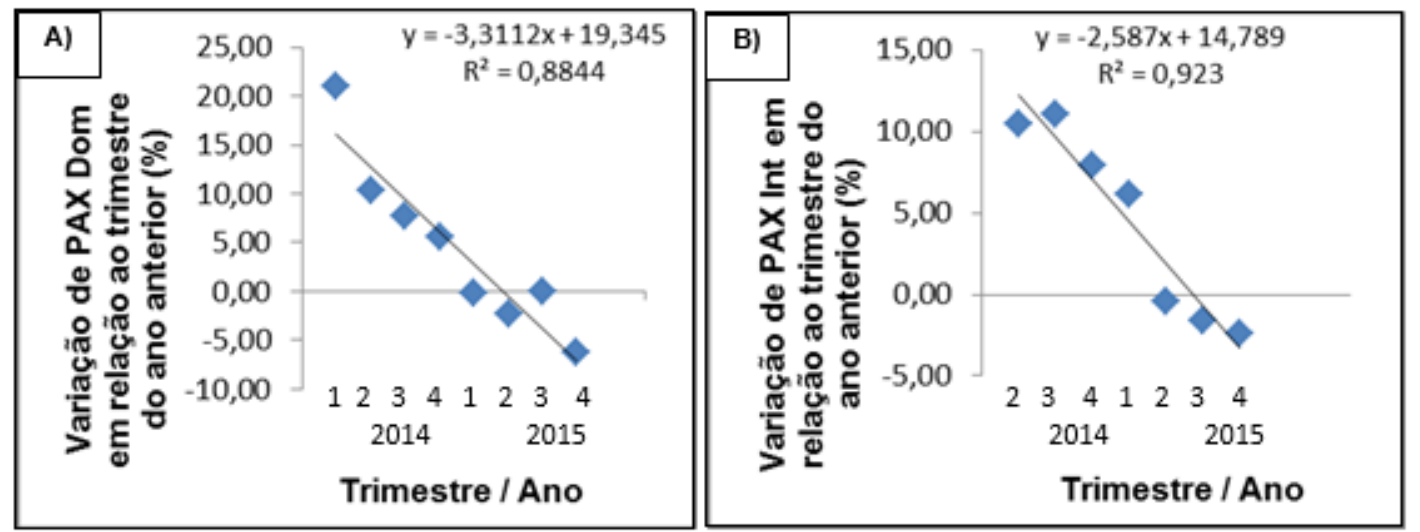

Figura 2. Movimentação de passageiros no transporte aéreo (PAX) A) doméstico (Dom) e B) internacional (Int) no Aeroporto Internacional de São Paulo/Guarulhos (GRU) entre 2014 e 2015, por trimestre (em relação ao mesmo trimestre do ano anterior) em relação ao trimestre/ano

Tabela 1: Equação da reta e fator de correlação $\left(R^{2}\right)$ da regressão linear dos dados de movimentação de passageiros aéreos entre 2013 e 2015 dos aeroportos analisados (continua)

\begin{tabular}{|c|c|c|c|}
\hline \multirow{7}{*}{$\begin{array}{l}\text { Aeroporto Internacional de São Paulo } \\
\text { (GRU) }\end{array}$} & Gráfico & Equação da reta & $\mathbf{R}^{\mathbf{2}}$ \\
\hline & PAX Dom por trimestre & $y=-3,3112 x+19,345$ & 0,8844 \\
\hline & PAX Int por trimestre & $y=-2,587 x+14,789$ & 0,9230 \\
\hline & PAX Dom versus PIB & $y=2,9676 x+9,9302$ & 0,8901 \\
\hline & $\mathrm{PAX}$ Int versus $\mathrm{PIB}$ & $y=2,6871 x+11,334$ & 0,8478 \\
\hline & PAX Dom versus Inflação & $y=-0,1805 x+11,449$ & 0,4080 \\
\hline & PAX Int versus Inflação & $y=-0,1029 x+8,8284$ & 0,2969 \\
\hline \multirow{7}{*}{$\begin{array}{l}\text { Aeroporto Internacional de Brasília } \\
\text { (BSB) }\end{array}$} & Gráfico & Equação da reta & $\mathbf{R}^{2}$ \\
\hline & PAX Dom por trimestre & $y=-0,6712 x+12,606$ & 0,1662 \\
\hline & PAX Int por trimestre & $y=-2,7947 x+29,646$ & 0,3205 \\
\hline & PAX Dom versus PIB & $y=0,8438 x+11,145$ & 0,3291 \\
\hline & $\mathrm{PAX}$ Int versus $\mathrm{PIB}$ & $y=-0,282 x+16,133$ & 0,0048 \\
\hline & PAX Dom versus Inflação & $y=-0,0223 x+10,452$ & 0,0285 \\
\hline & PAX Int versus Inflação & $y=-0,0805 x+21,899$ & 0,054 \\
\hline \multirow{7}{*}{$\begin{array}{l}\text { Aeroporto Internacional do Galeão } \\
\text { (GIG) }\end{array}$} & Gráfico & Equação da reta & $\mathbf{R}^{\mathbf{2}}$ \\
\hline & PAX Dom por trimestre & $y=-2,3483 x+12,011$ & 0,5546 \\
\hline & PAX Int por trimestre & $y=0,3709 x-1,5075$ & 0,0637 \\
\hline & PAX Dom versus PIB & $y=2,1702 x+5,455$ & 0,5935 \\
\hline & PAX Int versus PIB & $y=-1,131 x-3,4992$ & 0,4009 \\
\hline & PAX Dom versus Inflação & $y=-0,1474 x+7,1655$ & 0,3395 \\
\hline & PAX Int versus Inflação & $y=0,0486 x-2,0976$ & 0,2225 \\
\hline \multirow{7}{*}{$\begin{array}{l}\text { Aeroporto Internacional de Belém } \\
\text { (BEL) }\end{array}$} & Gráfico & Equação da reta & $\mathbf{R}^{2}$ \\
\hline & PAX Dom por trimestre & $y=-12,531 x+28,096$ & 0,7106 \\
\hline & PAX Int por trimestre & $y=-22,867 x+53,564$ & 0,7267 \\
\hline & PAX Dom versus PIB & $y=10,026 x-9,7599$ & 0,5701 \\
\hline & $\mathrm{PAX}$ Int versus $\mathrm{PIB}$ & $y=15,517 x-1,9316$ & 0,5479 \\
\hline & PAX Dom versus Inflação & $y=-0,9827 x+9,8501$ & 0,6788 \\
\hline & PAX Int versus Inflação & $y=-1,4622 x+24,468$ & 0,6045 \\
\hline \multirow{7}{*}{$\begin{array}{l}\text { Aeroporto Internacional de Salvador } \\
\text { (SSA) }\end{array}$} & Gráfico & Equação da reta & $\mathbf{R}^{\mathbf{2}}$ \\
\hline & PAX Dom por trimestre & $y=-2,2439 x+11,986$ & 0,4688 \\
\hline & PAX Int por trimestre & $y=3,6565 x-5,9364$ & 0,2470 \\
\hline & PAX Dom versus PIB & $y=2,2027 x+5,9605$ & 0,5661 \\
\hline & PAX Int versus PIB & $y=-4,6094 x-2,8261$ & 0,5540 \\
\hline & PAX Dom versus Inflação & $y=-0,1449 x+7,5118$ & 0,3035 \\
\hline & PAX Int versus Inflação & $y=0,4531 x-10,64$ & 0,7718 \\
\hline
\end{tabular}


Tabela 1: Equação da reta e fator de correlação $\left(R^{2}\right)$ da regressão linear dos dados de movimentação de passageiros aéreos entre 2013 e 2015 dos aeroportos analisados

\begin{tabular}{|c|c|c|c|}
\hline \multirow{7}{*}{$\begin{array}{l}\text { Aeroporto Internacional de Goiânia } \\
\text { (GYN) }\end{array}$} & Gráfico & Equação da reta & $\mathbf{R}^{2}$ \\
\hline & PAX Dom por trimestre & $y=-4,1184 x+25,184$ & 0,8775 \\
\hline & PAX Int por trimestre & - & - \\
\hline & PAX Dom versus PIB & $y=3,5367 x+13,188$ & 0,8108 \\
\hline & PAX Int versus PIB & - & - \\
\hline & PAX Dom versus Inflação & $y=-0,2251 x+15,39$ & 0,4073 \\
\hline & PAX Int versus Inflação & - & - \\
\hline \multirow{7}{*}{$\begin{array}{l}\text { Aeroporto Internacional de Porto Ale- } \\
\text { gre (POA) }\end{array}$} & Gráfico & Equação da reta & $\mathbf{R}^{\mathbf{2}}$ \\
\hline & PAX Dom por trimestre & $y=-1,5811 x+9,8165$ & 0,6698 \\
\hline & PAX Int por trimestre & $y=-10,155 x+41,504$ & 0,9345 \\
\hline & PAX Dom versus PIB & $y=1,5636 x+5,5916$ & 0,8207 \\
\hline & PAX Int versus PIB & $y=6,5022 x+15,346$ & 0,6499 \\
\hline & PAX Dom versus Inflação & $y=-0,0914 x+6,251$ & 0,3480 \\
\hline & PAX Int versus Inflação & $y=-0,4869 x+21,657$ & 0,4372 \\
\hline
\end{tabular}

Sendo Dom = Doméstico, Int = Internacional.

Assim, estes resultados estão coerentes com a crise econômica do Brasil no período. Apenas o Aeroporto Internacional de Salvador e o do Rio de Janeiro apresentaram aumento de movimentação de passageiros no transporte aéreo internacional no período analisado (coeficiente angular positivo para equação de PAX Int por trimestre).

Para verificar a relação entre a movimentação de passageiros no transporte aéreo doméstico e internacional e o PIB, a Figura 3 apresenta os gráficos e dados das regressões lineares realizadas para o Aeroporto Internacional de São Paulo/Guarulhos, e as equações obtidas são mostradas na Figura 3 e também na Tabela 1, que apresenta também as equações obtidas para os outros seis aeroportos estudados.

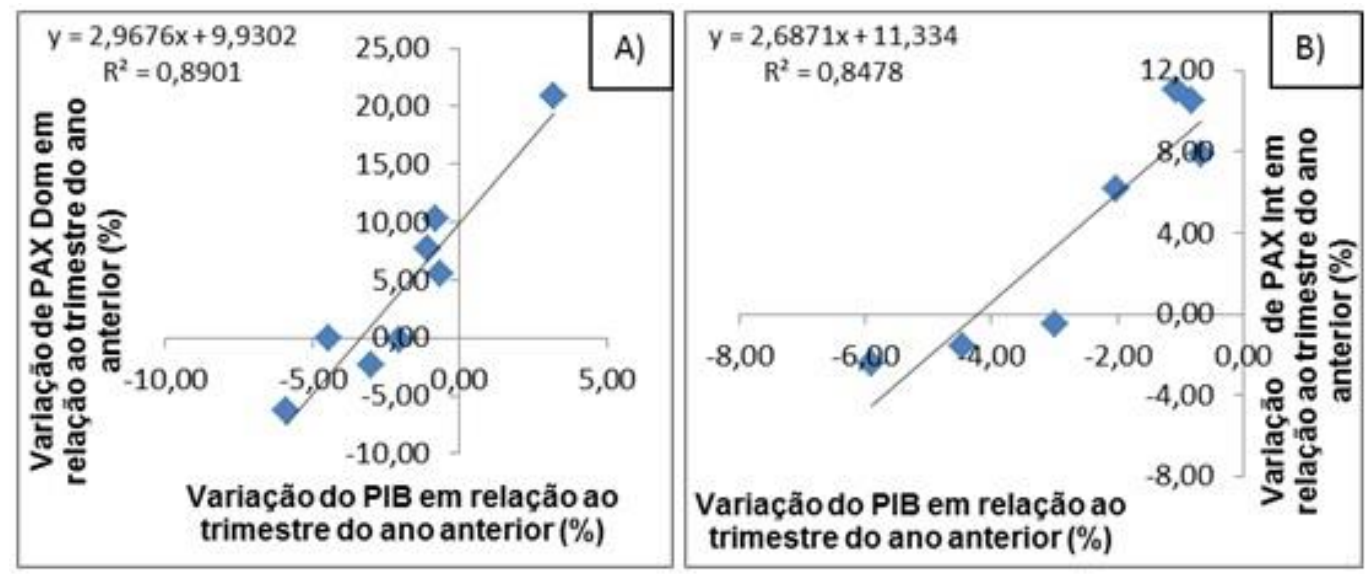

Figura 3. Movimentação de passageiros no transporte aéreo (PAX) A) doméstico (Dom) e B) internacional (Int) no Aeroporto Internacional de São Paulo/Guarulhos (GRU) entre 2014 e 2015, por trimestre (em relação ao mesmo trimestre do ano anterior) em relação à variação do PIB

A Figura 3 demonstra que o coeficiente de correlação está entre 0,8 e 0,9 para os dois gráficos, mostrando que as equações obtidas representam de forma adequada os dados coletados para o Aeroporto Internacional de São Paulo. A Tabela 1 apresenta coeficientes de correlação (equações para PAX Dom e PAX Int versus PIB) semelhantes ou um pouco menores para os outros aeroportos analisados. Um Aeroporto, porém, chama a atenção por apresentar coeficientes de correlação muito baixos: o Aeroporto Internacional de Brasília. Uma explicação para estes baixos valores de fator de correlação é o fato de o Aeroporto de Brasília estar se consolidando como o maior hub doméstico do Brasil, consequência direta do investimento de 1,2 bilhões de reais em obras de reforma e ampliação para a Copa do Mundo FIFA 2014. Além disso, Brasília é a capital do país e tem uma posição geográfica privilegiada, o que, associado ao investimento em infraestrutura que este aeroporto teve recentemente (até o ano de 2014), fez com que a movimentação de passageiros aéreos não diminuísse neste período de crise econômica do Brasil, TRANSPORTES | ISSN: 2237-1346 
ao contrário dos outros aeroportos estudados (Aeroporto de Brasília, 2014; Aeroporto de Brasília, 2015).

De acordo com a Figura 3 e a Tabela 1, o transporte aéreo doméstico mostrou maior fator de correlação do que o internacional para todos os aeroportos analisados, indicando que a crise econômica brasileira afetou o transporte aéreo doméstico em maior grau para os aeroportos estudados. Este resultado era esperado, uma vez que a crise financeira não ocorreu no mundo como um todo no período analisado, e, sendo assim, o transporte aéreo internacional deve sofrer menor influência da crise econômica brasileira do que o transporte aéreo doméstico. Porém, o transporte aéreo internacional também mostrou alto fator de correlação em relação ao PIB, também indicando estar relacionado com a crise financeira brasileira.

Já para a variável Inflação, as correlações com a movimentação de passageiros no transporte aéreo doméstico e internacional do Aeroporto Internacional de São Paulo são apresentadas na Figura 4, e as equações obtidas são apresentadas na Figura 4 e na Tabela 1, juntamente com as equações para os outros aeroportos.

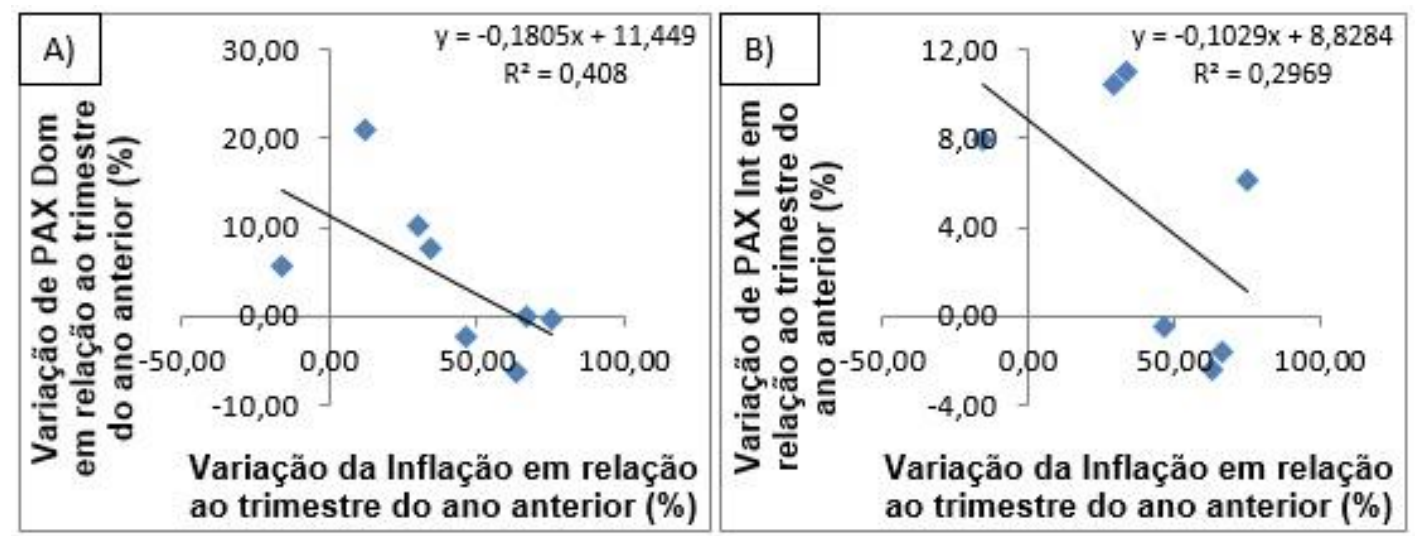

Figura 4. Movimentação de passageiros no transporte aéreo (PAX) A) doméstico (Dom) e B) internacional (Int) no Aeroporto Internacional de São Paulo (GRU) entre 2014 e 2015, por trimestre (em relação ao mesmo trimestre do ano anterior) em relação à variação da Inflação

Os valores de fator de correlação das equações obtidas após a regressão linear, conforme mostra a Figura 4 e a Tabela 1, são mais baixos para a variável Inflação do que quando a variável PIB foi utilizada para praticamente todos os aeroportos estudados. Caso seja retirado o ponto relativo ao quarto trimestre de 2014 (ponto discrepante da curva da Figura 1B), o fator de correlação das curvas da Figura 4 sobem de 0,4 para aproximadamente 0,8 para movimentação de passageiros domésticos e de aproximadamente 0,3 para 0,4 para movimentação de passageiros internacionais. Assim, em especial para movimentação de passageiros domésticos, a Inflação passa a ser uma boa variável para prever a movimentação de passageiros aéreos, embora a variável PIB ainda mostre maior precisão para a previsão (maior fator de correlação). Também para os demais aeroportos, mesmo desconsiderando este ponto, os coeficientes de correlação são maiores quando se utiliza o PIB como variável independente. Portanto, apesar de a Inflação ter grande potencial para ser utilizada como variável independente para a previsão de demanda de transporte aéreo, o PIB ainda apresenta melhores resultados neste tipo de estudo.

Diferentes estudos apontam fortes correlações entre PIB e movimentação de passageiros aéreos, em especial para períodos de longo prazo (Marazzo et al., 2010; Chi e Baek, 2013). Profillidis e Botzoris (2015), por exemplo, mostraram correlação entre a movimentação de passageiros aéreos em várias regiões do mundo entre 1980 e 2013, com valores de fator de correlação entre 0,75 e 0,99, dependendo da região analisada. Para curto prazo, entretanto, esta correlação não é tão direta (Hu et al, 2015), e, desta forma, menores valores de fator de correlação podem ser esperados. Assim, os valores de fator de correlação para as equações mostradas na Tabela 1, em especial para a utilização de PIB como variável 
independente, podem ser considerados bastante satisfatórios, uma vez que o período analisado é curto, já que o principal objetivo deste estudo é avaliar a influência da crise econômica do Brasil na movimentação de passageiros aéreos. Desta forma, foi analisado apenas um período específico.

Portanto, de acordo com a previsão do PIB brasileiro, é possível fazer uma previsão da demanda por transporte aéreo doméstico e internacional para cada um dos aeroportos aqui estudados, com exceção do Aeroporto de Brasília, que apresentou baixo fator de correlação para os dados estudados. De acordo com o Banco Central, a previsão do PIB para 2016 é de queda de 3,88 \% em relação ao ano de 2015. Os resultados encontrados, utilizando as equações mostradas na Tabela 1, são mostrados na Tabela 2 . Assim, com os dados de previsão da demanda por transporte aéreo mostrados na Tabela 2, é possível realizar o planejamento das necessidades de investimentos no setor em cada um dos aeroportos estudados. Para previsões futuras (2017, por exemplo), é possível utilizar as mesmas equações da Tabela 1 e, com a previsão do PIB para o ano em questão, pode-se obter as previsões para movimentação de passageiros no transporte doméstico e internacional para o ano analisado.

A Tabela 2 mostra previsões de demanda por transporte aéreo de acordo com as tendências apresentadas até aqui para os aeroportos estudados, com destaque para a redução de passageiros domésticos para a maior parte dos aeroportos no período analisado, sendo que o Aeroporto Internacional de Belém (BEL) foi o que mostrou maior queda de passageiros domésticos: cerca de $44 \%$ comparando a previsão para 2016 e os dados de 2015. Os dados refletem o período de crise econômica brasileira.

Tabela 2: Movimentação de passageiros no transporte aéreo doméstico e internacional de 2015 e previsão para 2016 para vários aeroportos do Brasil

\begin{tabular}{ccccc}
\hline \multirow{2}{*}{ Aeroporto } & \multicolumn{2}{c}{2015} & \multicolumn{2}{c}{ Previsão 2016 } \\
\cline { 2 - 5 } & PAX Doméstico & PAX Internacional & PAX Doméstico & PAX Internacional \\
\hline GRU & $25.365 .000^{\mathrm{a}}$ & $13.620 .000^{\mathrm{a}}$ & $25.339 .562^{\mathrm{d}}$ & $13.926 .668^{\mathrm{d}}$ \\
GIG & $12.840 .816^{\mathrm{b}}$ & $4.101 .413^{\mathrm{b}}$ & $12.599 .373^{\mathrm{d}}$ & $4.114 .684^{\mathrm{d}}$ \\
BEL & $2.951 .763^{\mathrm{c}}$ & $93.703^{\mathrm{c}}$ & $1.663 .384^{\mathrm{d}}$ & $42.748^{\mathrm{d}}$ \\
SSA & $8.302 .326^{\mathrm{c}}$ & $340.554^{\mathrm{c}}$ & $8.179 .068^{\mathrm{d}}$ & $383.987^{\mathrm{d}}$ \\
GYN & $3.193 .339^{\mathrm{c}}$ & - & $3.232 .743^{\mathrm{d}}$ & - \\
POA & $7.699 .115^{\mathrm{c}}$ & $495.06^{\mathrm{c}}$ & $7.722 .723^{\mathrm{d}}$ & $462.231^{\mathrm{d}}$ \\
\hline
\end{tabular}

a Fonte: Aeroporto Internacional de São Paulo/Guarulhos; ${ }^{b}$ Fonte: Aeroporto Internacional do Rio de Janeiro, c Fonte: Infraero, d Fonte: resultados da pesquisa.

Na maior parte dos estudos encontrados na literatura, o estudo de previsão de demanda no transporte aéreo é realizado de uma forma mais geral, como no estudo de Marazzo (2010), que estudou a movimentação de passageiros aéreos no Brasil como um todo, ou de forma mais específica e limitada, como no estudo de Scarpel (2013), que estudou a movimentação de passageiros de um aeroporto específico: o Aeroporto Internacional de São Paulo.

Já os resultados apresentados no presente estudo mostram um estudo mais amplo e equações distintas para a previsão da demanda por transporte aéreo para vários aeroportos do país, incluindo o principal aeroporto (Aeroporto Internacional de São Paulo) e também outros aeroportos espalhados ao longo das cinco regiões do Brasil (Norte, Nordeste, Sul, Sudeste, Centro-Oeste).

Por fim, para verificar o comportamento das empresas aéreas brasileiras frente à crise, a Figura 5 apresenta a variação do preço médio das tarifas aéreas domésticas no período e a quantidade de assentos ofertados, medidos em assentos quilômetros oferecidos (ASK).

De acordo com a Figura 5A, a partir do terceiro trimestre de 2014, as tarifas aéreas domésticas mostraram grande queda de preços, indicando uma tentativa das empresas aéreas em recuperar as vendas de passagens durante a crise financeira brasileira. Portanto, a queda na movimentação de passageiros no transporte aéreo doméstico durante o período de crise econômica do Brasil apresentada neste estudo poderia ter sido ainda maior caso as empresas aéreas não realizassem esta redução das tarifas aéreas no mesmo período. 


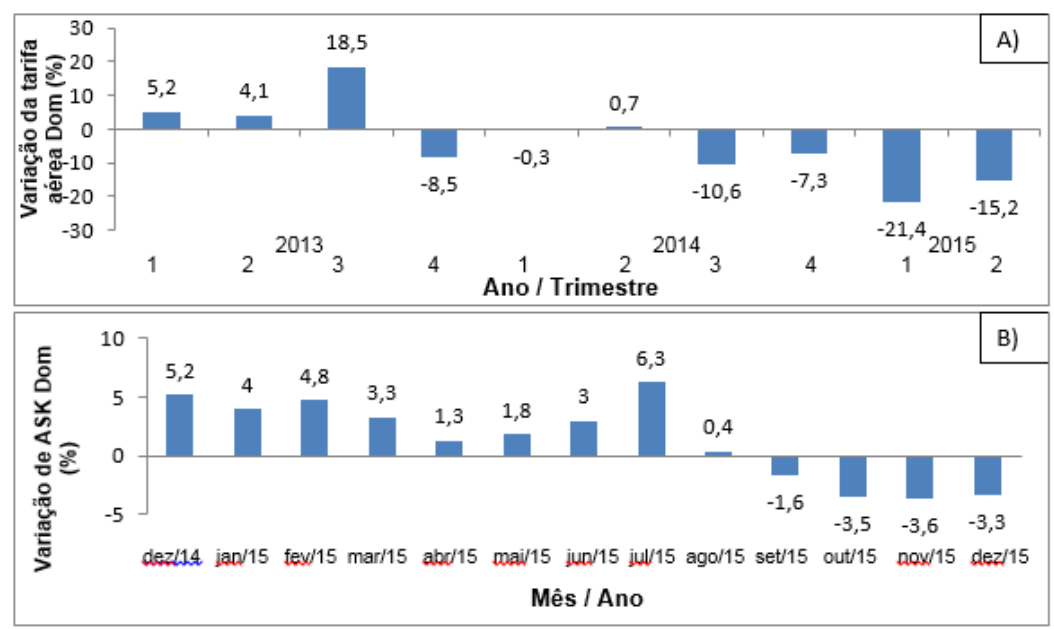

Figura 5. Dados do transporte aéreo doméstico (Dom) brasileiro para a variação da $A$ ) tarifa média real por trimestre e da B) quantidade de assentos ofertados (ASK) em relação ao mesmo período do ano anterior. Fonte: ANAC

Acompanhando a queda nas tarifas aéreas, também a quantidade de assentos ofertados no transporte aéreo doméstico brasileiro foi menor a partir de setembro de 2015, conforme mostra a Figura 5B. Desta forma, as empresas aéreas brasileiras enfrentaram a crise financeira no país reduzindo as tarifas do transporte aéreo e também reduzindo a quantidade de assentos ofertados no transporte aéreo doméstico.

\section{CONCLUSÕES}

Como foi observado ao longo do estudo, a movimentação de passageiros no transporte aéreo doméstico e internacional dos vários aeroportos do Brasil aqui estudados seguiu a mesma tendência entre $2013 \mathrm{e}$ 2015, que foi a de redução da movimentação, acompanhada da queda do PIB e aumento da Inflação no mesmo período, representando a crise econômica do Brasil. Um aeroporto que apresentou exceção a esta tendência foi o Aeroporto Internacional de Brasília, o que foi explicado pela característica deste aeroporto de estar se consolidando como o maior hub doméstico do Brasil.

Após a realização de regressão linear, a movimentação de passageiros no transporte aéreo doméstico mostrou melhor ajuste das equações do que a movimentação no transporte aéreo internacional, indicando que a crise econômica brasileira influenciou em maior grau o transporte aéreo doméstico.

Também foi realizada regressão linear dos dados de movimentação de passageiros aéreos versus PIB e versus Inflação. Quando o PIB foi utilizado como variável independente, os valores de fator de correlação foram maiores do que para a Inflação. Desta forma, o PIB foi utilizado como variável independente para as previsões de demanda da movimentação de passageiros para o ano de 2016 para os vários aeroportos aqui estudados. Estas previsões são fundamentais para o planejamento do setor aéreo de cada um dos aeroportos estudados.

O comportamento das empresas aéreas brasileiras frente à crise econômica do país também foi estudado, sendo que redução da movimentação de passageiros no transporte aéreo durante o período analisado foi acompanhada de redução nas tarifas aéreas, assim como redução na quantidade de assentos ofertados no transporte aéreo doméstico.

Uma sugestão para estudos futuros é expandir o estudo para outros aeroportos do país, além de estudar outros modelos de regressão para analisar a influência da crise econômica na movimentação de passageiros aéreos.

\section{AGRADECIMENTOS}

Ao Conselho Nacional de Desenvolvimento Científico e Tecnológico - CNPq (Processo: 160348/2015-3, PDJ) e à Fundação de Amparo à Pesquisa do Estado de Goiás (FAPEG). 


\section{REFERÊNCIAS}

Aeroporto de Brasília, 2014. Aeroporto de Brasília é o maior hub doméstico do país. http://www.bsb.aero

Aeroporto de Brasília, 2015. Aeroporto de Brasília mantém crescimento e movimentação média diária sobe até $8 \%$. http://www.bsb.aero.

ANAC, 2016. Tarifas Aéreas Domésticas. www.anac.gov.br

Ashley, D.J.; Hanson, P.; Veldhuis, J. A policy-sensitive traffic forecasting model for Schiphol Airport. Journal of Air Transport Management, v. 2, p. 89-97, 1995. DOI: 10.1016/0969-6997(95)00034-8.

Chi, J. e Baek, J (2013) Dynamic relationship between air transport demand and economic growth in the United States: a new look. Transport Policy, v. 29, p. 257-260. DOI: 10.1016/j.tranpol.2013.03.005.

Dobruszkes, F. e Van Hamme, G (2011) The impact of the current economic crisis on the geography of air traffic volumes: an empirical analysis. Journal of Transport Geography, v. 19, p. 1387-1398. DOI: 10.1016/j.jtrangeo.2011.07.015.

$\mathrm{Hu}$, Y. et al. (2015). Domestic air passenger traffic and economic growth in China: Evidence from heterogeneous panel models. Journal of Air Transport Management, v. 42, p. 95-100. DOI: 10.1016/j.jairtraman.2014.09.003.

IBGE, 2014. http://www.ibge.gov.br

ICAO, 2006. Manual on Air Traffic Forecasting, third ed.

Kalayci, S.; Yanginlar, G. The Effects of Economic Growth and Foreign Direct Investment on Air Transportation: Evidence from Turkey. International Business Research, v. 9, p. 154-162, 2016. DOI: http://dx.doi.org/10.5539/ibr.v9n3p154.

Marazzo, M.; Scherre, R. e Fernandes, E (2010) Air transport demand and economic growth in Brazil: A time series analysis. Transportation Research Part E: Logistics and Transportation Review, v. 46, p. 261-269. DOI: 10.1016/j.tre.2009.08.008.

Profillidis, V. e Botzoris, G (2015) Air passenger transport and economic activity. Journal of Air Transport Management, v. 49, p. 23-27. DOI: 10.1016/j.jairtraman.2015.07.002.

Rimmer, P. J (2000) Effects of the Asian Crisis on the geography of Southeast Asia's air traffic. Journal of Transport Geography, v. 8, p. 83-97. DOI: 10.1016/S0966-6923(99)00041-1.

Scarpel, R. A (2013) Forecasting air passengers at São Paulo International Airport using a mixture of local experts. Journal of Air Transport Management, v. 26, p. 35-39. D0I:10.1016/j.jairtraman.2012.10.001.

Sivrikaya, O. e Tunç, E. Demand Forecasting for Domestic Air Transportation in Turkey. The Open Transportation Journal, v. 7, p. 20-26, 2013. DOI: 10.2174/1874447820130508001. Portal da Transparência. http://www.portaltransparencia.gov.br

Vasconcellos, M. A. S. e Garcia, M. E (1998) Fundamentos de Economia. Editora Saraiva, São Paulo.

Vega, A. e Reynolds-Feighan, A. The impact of the great recession on Irish air travel: An intermodal accessibility analysis. Journal of Air Transport Management, v. 51, p. 1-18, 2016. DOI: 10.1016/j.jairtraman.2015.11.002.

Wadud, Z. The asymmetric effects of income and fuel price on air transport demand. Transportation Research Part A: Policy and Practice, v. 65, p. 92-102, 2014. DOI: 10.1016/j.tra.2014.04.001.

Wadud, Z. Imperfect reversibility of air transport demand: Effects of air fare, fuel prices and price transmission. Transportation Research Part A, v. 72, p. 16-26, 2015. DOI: 10.1016/j.tra.2014.11.005.

Wu, C.; Han, J.; Hayashi, Y. Airport attractiveness analysis through a gravity model: A case study of Chubu International Airport in Japan. Eastern Asia Soc. Transp. Stud., v. 8, 2011. DOI: Http://doi.org/10.11175/eastpro.2011.0.419.0. 